\title{
Initial Ionization Rates in Shock-Heated Argon, Krypton, and Xenon
}

\author{
Kenneth E. Harwell* and Robert G. Jahn $\dagger$ \\ Daniel and Florence Guggenheim Jet Propulsion Center, \\ California Institute of Technclogy, Pasadena, California \\ (Received 18 August 1963; revised manuscript received 11 September 1963)
}

\begin{abstract}
The rate of ionization behind strong shock waves in argon, krypton, and xenon, is observed by a transverse microwave probe, over a range of electron densities low enough that atom-atom inelastic collisions are the rate-determining mechanism. Shocks of Mach number 7.0 to 10.0 propagate down a 2-in. sq. aluminum shock tube into ambient gases at pressures of 3.0 to $17.0 \mathrm{~mm}$. $\mathrm{Hg}$., heating them abruptly to atomic temperatures of $5500^{\circ} \mathrm{K}$ to $9600^{\circ} \mathrm{K}$. The subsequent relaxation toward ionization equilibrium is examined in its early stages by the reflection, transmission, and phase shifts of a $24.0 \mathrm{Gc} / \mathrm{sec}(1.25 \mathrm{~cm})$ transverse microwave beam propagating between two rectangular horns abreast a glass test section. The data yield effective activation energies of $11.9 \pm 0.5 \mathrm{eV}$ for argon, $10.4 \pm 0.5 \mathrm{eV}$ for krypton, and $8.6 \pm 0.5 \mathrm{eV}$ for xenon. These coincide, within experimental error, with the first excitation potentials, rather than the ionization potentials of the gases, indicating that in this range ionization proceeds via a two-step process involving the first excited electronic states of which the excitation step is rate controlling.
\end{abstract}

\section{INTRODUCTION}

$\mathrm{T}$ HE purpose of the experiment reported here is to study the initial ionization of shock-heated argon, krypton, and xenon. "Initial" ionization refers to that regime of very low ionization, proposed by Bond $^{1}$ and others, wherein the relatively efficient electron-atom collision processes are so rare that the gas must have recourse to much less effective heavy particle collisions to generate its first few ions. A variety of reaction schemes can be proposed for this regime. For example, in pure gases, the ionization may be achieved by single atom-atom collisions:

$$
\mathrm{A}+\mathrm{A} \stackrel{k_{1}}{\rightarrow} \mathrm{A}^{+}+e+\mathrm{A},
$$

or by two successive atom-atom collisions, involving an intermediate excited state, $\mathrm{A}^{*}$ :

$$
\begin{gathered}
\mathrm{A}+\mathrm{A} \underset{k^{*}}{\stackrel{k^{*}}{\rightleftharpoons}} \mathrm{A}^{*}+\mathrm{A}, \\
\mathrm{A}+\mathrm{A}^{*} \stackrel{k_{+}}{\longrightarrow} \mathrm{A}^{+}+e+\mathrm{A}, \\
{\left[\mathrm{A}^{*} \stackrel{\vec{k}_{\nu}}{\rightleftharpoons} \mathrm{A}+h_{\nu}\right] .}
\end{gathered}
$$

Here $k_{1}, k_{*}, k_{*}^{\prime}, k_{+}$symbolize reaction rates in the usual chemical sense, and $\bar{k}_{v}$ is a composite rate discussed below. Because of the relatively high atomic levels and small collision cross sections of these pure gas "reactions," a very small amount of impurity may participate significantly, either by directly ionizing the test gas:

\footnotetext{
* Present address: Department of Aerospace Engineering, Auburn University, Auburn, Alabama.

$\dagger$ Present address: Department of Aeronautical Engineering, Princeton University, Princeton, New Jersey.

${ }^{1}$ J. W. Bond, Phys. Rev. 105, 1683 (1957).
}

$$
\mathrm{I}+\mathrm{A} \rightarrow \mathrm{A}^{+}+e+\mathrm{I},
$$

or, more likely, by becoming ionized itself, and possibly undergoing subsequent charge exchange with the test gas:

$$
\begin{gathered}
\mathrm{A}+\mathrm{I} \rightarrow \mathrm{I}^{+}+\mathrm{A}+e \\
\mathrm{I}^{+}+\mathrm{A} \rightarrow \mathrm{A}^{+}+\mathrm{I} .
\end{gathered}
$$

The impurity particle involved, I, may be either in the gas or on the wall of the container.

The theoretical formulation of any of these reactions is quite similar. For example, the frequency with which process (1) proceeds in a gas whose atoms are in Maxwellian equilibrium may be written ${ }^{2}$ :

$$
\begin{array}{r}
\frac{d}{d t}\left(N_{+}\right)_{1}=\frac{1}{2} N^{2}\left(\frac{M}{2 \pi K T}\right)^{3} \int_{0}^{\infty} e^{-M v_{0}^{2} / K T} 4 \pi v_{0}^{2} d v_{0} \\
\cdot \int_{0_{0}}^{\infty} e^{-M g^{2} / 4 K^{T}} Q_{1}(g) 4 \pi g^{3} d g \equiv k_{1}(T) N^{2},
\end{array}
$$

where $N, M$, and $T$ are the atomic particle density, mass, and temperature, $K$ is Boltzmann's constant, $v_{0}$ is the center of mass speed of two colliding atoms, and $g$ their relative speed, which has a minimum value $g_{0}$ corresponding to the threshold energy for ionization, $E_{\mathrm{i}}$

$$
g_{0}=\left(4 E_{\mathrm{i}} / M\right)^{\frac{1}{2}} \text {. }
$$

The magnitude of $d\left(N_{+}\right)_{1} / d t$ hinges on the dependence of the ionization cross section, $Q_{1}$, on the relative particle speed at impact. Weymann ${ }^{3}$ assumes

${ }^{2} \mathrm{~S}$. Chapman and T. G. Cowling, The Mathematical Theory of Non-Uniform Gases (Cambridge University Press, London, 1952), chap. V.

${ }^{3}$ H. D. Weymann, University of Maryland Technical Note BN-144, 1958. 
$Q_{1}$ is constant from $g_{0}$ to $\infty$ and obtains

$$
\frac{d}{d t}\left(N_{+}\right)_{1}=2 N^{2} Q_{1}\left(\frac{K T}{\pi M}\right)^{\frac{1}{2}}\left(\frac{E_{\mathrm{i}}}{K T}+1\right) e^{-E_{\mathrm{i} / K T}} .
$$

However, most inelastic collision cross sections have energy dependence rising steeply from threshold. Since we will be concerned with a situation where $K T \ll E_{\mathrm{i}}$ it seems more reasonable to approximate $Q_{1}$ by a linear dependence on energy (transferred) above threshold:

$$
Q_{1}=C_{1}\left(E-E_{\mathrm{i}}\right)=\frac{1}{4} M C_{1}\left(g^{2}-g_{0}^{2}\right),
$$

in terms of which the ionization frequency becomes:

$\frac{d}{d t}\left(N_{+}\right)_{1}=4 C_{1}(\pi M)^{-\frac{1}{2}} N^{2}(K T)^{\frac{3}{2}}\left(\frac{E_{\mathrm{i}}}{2 K T}+1\right) e^{-E_{i} / K T}$.

Taking the natural logarithm, and differentiating with respect to $1 / K T$ yields

$$
\begin{aligned}
& \frac{d\left[\ln \frac{d}{d t}\left(N_{+}\right)_{1}\right]}{d(1 / K T)} \\
& \quad=-E_{\mathrm{i}}\left[1+\frac{3}{2} \frac{K T}{E_{\mathrm{i}}}-\frac{1}{E_{\mathrm{i}} / K T+2}\right] .
\end{aligned}
$$

For $K T \ll E_{\mathrm{i}}$

$$
\frac{d\left[\ln \frac{d}{d t}\left(N_{+}\right)_{1}\right]}{d(1 / K T)} \approx-E_{\mathrm{i}},
$$

expressing the familiar result that the slope of a graph of the logarithm of the rate of ionization, $\ln d / d t\left(N_{+}\right)$, vs $1 / K T$ is approximately the activation energy for the process being considered, $E_{\mathrm{i}}$, in this case the ionization potential.

An identical relation prevails for the first step of reaction (2), except that the threshold energy is now the excitation potential, $E_{*}$, and the cross section involved is that for excitation to the excited state $\mathrm{A}^{*}$ :

$$
Q_{*}=C_{*}\left(E-E_{*}\right) .
$$

The second step, (2b) would be similarly described in terms of a threshold energy, $E_{\mathrm{i}}-E_{*}$, and a cross section for ionization from the excited state

$$
Q_{+}=C_{+}\left[E-\left(E_{\mathrm{i}}-E_{*}\right)\right] \text {. }
$$

The effective activation energy, the density dependence, and the relative efficiency of the composite two-step process all depend strongly on the lifetime of the excited state, $\mathrm{A}^{*}$, in comparison to the mean free time for ionization by process $(2 \mathrm{~b})$.
Since for the noble gases even the lowest excited states have energies well above half the ionization potentials, the energy increments involved in the second step, $\left(E_{\mathrm{i}}-E_{*}\right)$ will be substantially less than those for the first step, $E_{*}$. Also, the geometrical "size" of an excited noble gas atom presumably exceeds that of its ground state, and a continuum of final states is available for the second step compared to a discrete level for the first. It is thus reasonable to anticipate significantly higher collision efficiencies for the second step, i.e., $k_{+} \gg k_{*}$.

The efficiency of the two-step process thus hinges on the level of "steady state" concentration of A* which can be sustained. This in turn depends on the relative rates of collisional de-excitation $\left(k_{*}^{\prime}\right)$, and radiative decay $\left(\bar{k}_{v}\right)$, to the ionization step $\left(k_{+}\right)$. The natural lifetime of a normal excited state against radiative decay $\left(\sim 10^{-8} \mathrm{sec}\right)$ is probably inadequate to support this process. However, the gas could possibly make use of a longer-lived metastable level, or of a resonance trapping mechanism $^{3.4}$ wherein the photon emitted on radiative decay is captured and re-emitted many times by adjacent atoms before escaping from the gas. In this way the effective lifetime of the excited state would be substantially protracted. In either case, the process is symbolized by equation (2c) where $\bar{k}_{\nu}$ represents the effective reaction rate coefficient for decay of $\mathrm{A}^{*}$ by spontaneous radiation, and may depend on the gas density and total sample size, as well as the temperature.

To outline the various possible ionization profiles, consider the kinetic rate equations appropriate to the "reactions" (2a)-(2c):

$$
\begin{gathered}
\frac{d}{d t}\left(N_{*}\right)=k_{*} N^{2}-\left(k_{+}+k_{*}^{\prime}\right) N N_{*}-\overline{k_{\nu}} N_{*}, \\
\frac{d}{d t}\left(N_{+}\right)=k_{+} N N_{*} .
\end{gathered}
$$

For the low-level regime, $N$ greatly exceeds $N_{*}$ and $N_{+}$and may be regarded as constant here. The solution to (14) is then

$$
\begin{gathered}
N_{*}=k_{*} \tau N^{2}\left(1-e^{-t / \tau}\right), \\
\tau=\left[\left(k_{+}+k_{*}^{\prime}\right) N+\bar{k}_{\nu}\right]^{-1} .
\end{gathered}
$$

and thus the build-up of ion density by this mechanism becomes

$$
N_{+}=k_{*} k_{+} \tau N^{3}\left[t-\tau\left(1-e^{-t / \tau}\right)\right] .
$$

4 A. C. G. Mitchell and M. W. Zemansky, Resonance Radiation and Excited Atoms (Cambridge University Press, New York, 1961). 
TABle I. Characteristics of various ionization routes in the atom-atom regime.

\begin{tabular}{|c|c|c|c|c|c|c|}
\hline PROCESS & \begin{tabular}{|l|} 
COLLLISIONAL \\
DE-EXCIT.
\end{tabular} & $\begin{array}{c}\text { RAD. } \\
\text { DECAY }\end{array}$ & PHASE & $\begin{array}{l}\text { DENSITY } \\
\text { ORDER }\end{array}$ & $\begin{array}{l}\text { TIME } \\
\text { DEP. }\end{array}$ & $\begin{array}{c}\text { ACT. ENERGY } \\
\left(k T<<E_{*}\right)\end{array}$ \\
\hline \multirow[t]{6}{*}{ 2-STEP } & \multirow{4}{*}{$k_{*}^{\prime}<<k_{+}$} & \multirow[t]{2}{*}{$\bar{k}_{v}<<k_{t} N$} & $t<\frac{1}{k_{+} N}$ & $N^{3}$ & $t^{2}$ & $E_{i}$ \\
\hline & & & $t>>\frac{1}{k_{+} N}$ & $N^{2}$ & $t$ & $E_{*}$ \\
\hline & & \multirow[t]{2}{*}{$\bar{k}_{v}>>k_{+} N$} & $t<\frac{1}{\bar{k}_{v}}$ & $N^{3}$ & $t^{2}$ & $E_{i}$ \\
\hline & & & $t>>\frac{1}{\bar{k}_{v}}$ & $N^{3}$ & $t$ & $E_{i}$ \\
\hline & \multirow[t]{2}{*}{$k_{*}^{\prime}>>k_{+}$} & \multirow[t]{2}{*}{$\bar{k}_{v}<k_{*}^{\prime} N$} & $t<<\frac{1}{k_{*}^{\prime} N}$ & $N^{3}$ & $t^{2}$ & $E_{i}$ \\
\hline & & & $1>>\frac{1}{k_{*}^{\prime} N}$ & $\overline{N^{2}}$ & $t$ & $E_{i}$ \\
\hline I-STEP & $\longrightarrow$ & $\longrightarrow$ & - & $\mathrm{N}^{2}$ & $t$ & $E_{i}$ \\
\hline
\end{tabular}

Suppose first that collisional deexcitation is negligible, and that the resonance radiation is effectively trapped, or that the excited state is metastable, i.e., $k_{*}^{\prime} \ll k_{+}$, and $\bar{k}_{v} \ll k_{+} N$. Then,

$$
N_{+} \approx k_{*} N^{2}\left[t-\left(1 / k_{+} N\right)\left(1-e^{-k_{+} N t}\right)\right] .
$$

In this case, for times up to $t \approx 1 / k_{+} N$, the reaction is in an inception phase, building up the $A^{*}$ population to some steady state level, after which ions are generated at a constant rate

$$
\begin{aligned}
& \frac{d}{d t}\left(N_{+}\right) \rightarrow k_{*} N \\
& \quad=4 C_{*} N^{2}(K T)^{\frac{3}{2}}(\pi M)^{-\frac{1}{2}}\left(\frac{E_{*}}{2 K T}+1\right) e^{-E * / K T},
\end{aligned}
$$

which is completely characterized by the threshold energy, cross section, and density dependence of the excitation reaction, Eq. (2a).

As a second possibility, suppose collisional deexcitation remains negligible, but the radiative decay restrains the $A^{*}$ density to a low enough level to impede the reaction, i.e., $\bar{k}_{v} \gg k_{+} N$. Then

$$
N_{+} \approx \frac{k_{*} k_{+}}{\bar{k}_{\nu}} N^{3}\left[t-\frac{1}{\bar{k}_{\nu}}\left(1-e^{-\bar{k}_{\nu} t}\right)\right] .
$$

In this case, the inception phase lasts for a shorter time $t \approx 1 / \bar{k}_{v} \ll 1 / k_{+} N$ and is again followed by a linear phase, proceeding more slowly than (19), and third order in neutral density:

$$
\begin{aligned}
& \frac{d}{d t}\left(N_{+}\right) \rightarrow \frac{k_{*} k_{+}}{\bar{k}_{v}} N^{3} \\
& \quad \sim N^{3}\left(\frac{E_{*}}{2 K T}+1\right)\left(\frac{E_{\mathrm{i}}-E_{*}}{2 K T}+1\right) e^{-E_{\mathrm{i}} / K^{T}} .
\end{aligned}
$$

If collisional deexcitation were the dominant effect in establishing the steady state concentration of $\mathrm{A}^{*}$, i.e., $k_{*}^{\prime} \gg k_{+} ; k_{*}^{\prime} N \gg \bar{k}_{\nu}$, then

$$
N_{+} \approx \frac{k_{*} k_{+}}{k_{*}^{\prime}} N^{2}\left[t-\frac{1}{k_{*}^{\prime} N}\left(1-e^{-k_{*}^{\prime} N t}\right)\right] .
$$

Here the inception phase is again shorter: $t \approx$ $1 / k_{*}^{\prime} N \ll 1 / \bar{k}_{\nu}$, and is again followed by a linear phase, quadratic in neutral density.

These various regimes are summarized in Table I, in comparison with the single-step reaction (1).

The primary purpose of the experiment described is to measure the atom-atom ionization rates of various noble gases over a range of atomic temperatures and densities, and thereby to attempt to distinguish among the various mechanisms outlined above. To accomplish this purpose, it clearly is necessary to insure that the impurity reactions, (3) and (4), and all electron-atom ionization reactions are rendered insignificant. Impurity reactions are well known to be troublesome in such studies, ${ }^{5}$ as could be anticipated from the comparatively low excitation and ionization potentials available in many metal and molecular vapors. It is found empirically in the course of the experiments described here that impurity levels above one part per million significantly bias the results. Impurity levels above ten parts per million completely obscure the reactions of interest.

The suppression of electron-atom reactions dictates an upper limit on the ionization density which can be tolerated. An analysis similar to that above yields an ionization rate for direct electron-atom ionization $^{5}$

$\frac{d}{d t}\left(N_{+}\right)_{\mathrm{e}}=4 C_{\mathrm{e}}\left(\frac{2}{\pi m}\right)^{\frac{1}{2}} N n\left(K T_{\mathrm{e}}\right)^{\frac{3}{2}}\left(\frac{E_{\mathrm{i}}}{2 K T_{\mathrm{e}}}+1\right) e^{-E_{\mathrm{i}} / K T_{\mathrm{e}}}$,

${ }^{5}$ H. E. Petschek and S. Byron, Ann. Phys. (N. Y.) 1, 270 (1957). 
where $n, m$, and $T_{\mathrm{e}}$ are the free electron number density, mass, and temperature, and $C_{\mathrm{e}}$ is the cross section coefficient above threshold

$$
Q_{\mathrm{e}}=C_{\mathrm{e}}\left(E-E_{\mathrm{i}}\right) .
$$

Similar relations could again be expressed for the two-step electron-atom processes. If it is assumed that as the ion density grows, the first electronatom process to compete successfully with the prevailing atom-atom process involves the same type of reaction route as the latter, then the break-even level should be defined, for $T^{r}=T_{\mathrm{e}}$ by:

$$
(2 M / m)^{\frac{1}{2}} n C_{\mathrm{e}}=N C_{A}
$$

where $C_{\mathrm{e}}$ and $C_{A}$ here denote the cross section coefficients for the prevailing processes. Even if these specific processes were known, accurate numerical values for the coefficients would not be available. The existing data ${ }^{5-7}$ indicates $C_{A}>(0.01) C_{\mathrm{e}}$ is a reasonable assumption, on which basis the breakeven ionization level is at least $10^{-5}$, a value quite conservative compared with the conventional equivalent velocity hypothesis.

The desired experiments therefore must provide samples of very pure gas of constant (translational) temperatures in which ionization proceeds at a level below $10^{-5}$, at a fast enough rate, yet for a long enough time that the rate can be precisely determined. Shock waves of Mach number range 5 to 10 propagating into monatomic gases at a few $\mathrm{mm} \mathrm{Hg}$ ambient pressure in a shock tube, establish a few hundred microseconds of nearly isothermal flow at total densities $\approx 10^{18} \mathrm{~cm}^{-3}$. A diagnostic device sensitive to free electron densities below $10^{13} \mathrm{~cm}^{-3}$ is thus required, with adequate spatial and temporal resolution to follow the axial gradients established by the prevailing ionization reaction rate in this zone of gas. The choice was a $1.25 \mathrm{~cm}$ wavelength transverse microwave probe, described in detail elsewhere. ${ }^{8}$

\section{APPARATUS AND PROCEDURE}

The shock tube employed was specifically designed to combine the necessary ruggedness for high shock strength operation with a reasonable level of cleanliness. The major portion of the tube was assembled from lengths of cold-extruded, heavywall aluminum pipe (6066 - T5), having an interior

${ }^{6}$ H. S. W. Massey and E. H. S. Burhop, Electronic and Ionic Impact Phenomena (Oxford University Press, London, 1952).

7 A. von Engel, Ionized Gases (Oxford University Press, London, 1955).

${ }^{8}$ R. G. Jahn, Phys. Fluids 5, 678 (1962).

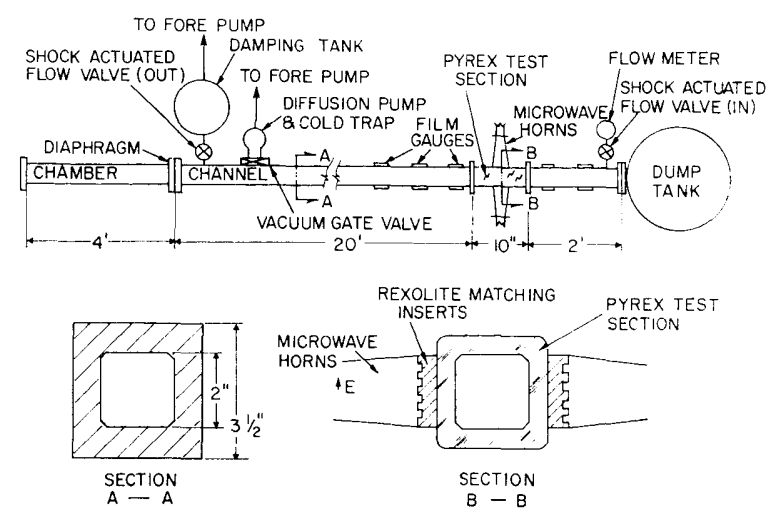

FIG. 1. Schematic diagram of shock tube.

cross section 2 -in. sq., with $\frac{5}{32}$-in. flat corner fillets, and a $\frac{3}{4}$ inch wall thickness (cf. Fig. 1). ${ }^{9}$ A rectangular channel was thus provided without the need for longitudinal seams. The lengths of tubing were joined with simple "O-ring" butt joints, constrained by split-ring flanges. ${ }^{10}$ Access ports to the tube were restricted to a minimum number and all were covered with cylindrical, "O-ring" flange fittings.

The test section was a 10 -in length of heavy wall, precision bore, square pyrex pipe, ${ }^{11}$ whose interior surface, hot-formed over a stainless steel mandril, precisely matched the interior dimensions of the adjacent aluminum sections of the shock tube. This pyrex section was attached to the tube, $20 \mathrm{ft}$ from the diaphragm, by means of a heavy steel yoke which permitted precise alignment of the inner walls at the junctions with the aluminum sections. Two feet beyond the test section, the tube terminated into a $10 \mathrm{ft}^{3}$ stainless steel dump tank, needed to reduce the final pressure in the tube to a level tolerable to the glass pipe.

The desired shock strengths were generated by pressure bursting diagonally-scribed aluminum and copper diaphragms of various thicknesses by various types and pressures of driver gas. Hydrogen up to 1000 psi was needed to generate the strongest shocks in argon. Helium was used for the weaker shocks in argon, and for all of the shock strengths generated in krypton. Various helium-argon mixtures were used as drivers for xenon.

Thin platinum film gauges, mounted on small glass plugs, recorded the time of passage of the

9 The authors are indebted to Mr. W. Thornton of the Alcoa Company for his personal interest in the preparation of this unusual extrusion.

${ }_{10}$ Professor D. Morelli and his students designed and implemented the mechanical details of the tube and its suspension.

${ }^{11}$ Fischer-Porter Company. 


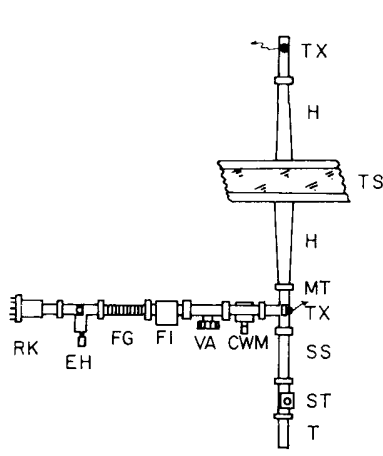

(a)

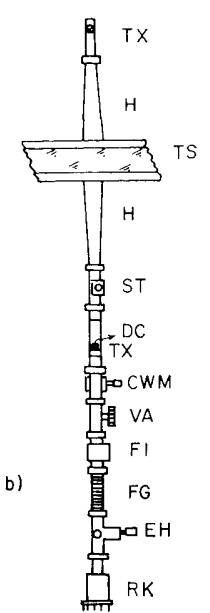

FIG. 2. Two microwave circuits for transverse probe: (a) reflection detector on magic tee; (b) reflection detector on directional coupler. Notation: RK, reflex klystron; $\mathrm{EH}, E$ and $H$ plane tuner; FG, section of flexible waveguide; FI, ferrite isolator; VA, variable attenuator; CWM, cavity wave meter (absorption type); T, termination; ST, stub tuner; SS, straight section; TX, tunable crystal mount; $\mathrm{H}$, horn MT, magic tee; DC, directional coupler; TS, test section (pyrex).

shock over given positions along the tube. The amplified responses of two platinum film gauges adjacent to the test section, fed into a Berkeley 7260 time interval crystal counter, yielded the shock Mach number at the test section to better than $1 \%$. The history of shock attenuation during its propagation down the tube was likewise determined by displaying the signals from a series of several film gauges directly onto a Tektronix 551 dual beam oscilloscope.

Largely by trial and error, the absolute leak rate of the tube and its associated gas handling equipment was reduced to less than $5 \times 10^{-4} \mu / \mathrm{min}$, which was felt to be adequate in comparison with the outgassing rate of about $2 \times 10^{-2} \mu / \mathrm{min}$ which prevailed even after heating the entire tube to $100^{\circ} \mathrm{C}$ for several hours. To combat the introduction of impurities via the outgassing, the test gas was allowed to flow continuously down the shock tube from a valve near the mouth of the dump tank, to a valve near the diaphragm, at rates as high as 1000 times the outgassing. These valves remained open until slammed shut by the passage of the shock wave over their ports. 'The ultimate impurity level prevailing in the gas at the time of passage of the shock front was reducible by this technique to about 1 part in $10^{7}$.

The build-up of ionization density in the gas behind the shock front was observed by a transverse microwave probe, described in detail elsewhere. ${ }^{s}$ Briefly, a pair of rectangular horns face each other across the glass test section, matched to it and to each other with a VSWR of about 1.01. These are driven at $24.0 \mathrm{Gc} / \mathrm{sec}$ by one of two circuits of standard K-band components; one involving a directional coupler, the other a magic tee, as shown in Fig. 2.

Using these microwave circuits, the transmitted power amplitude, the reflected power amplitude, and the transmission phase were recorded as functions of time after passage of the shock wave, on a dual-beam Tektronix oscilloscope. A typical record is shown in Fig. 3. Using the process outlined in Ref. 8, the electron density time history $n(t)$ and the collision frequency $\nu_{c}$ were then determined from this data.

Corrections were applied to the raw microwave data for nonlinearity of the crystal detectors, refraction of the radiation patterns, and departures from the theoretically assumed plane slab geometry of the ionized gas sample, wherever needed, in accordance with calibration measurements and theoretical models described in Ref. 8. In addition, substantial corrections were made to compensate for the measured attenuation of the shock fronts. A shock varying in strength during its propagation down the tube leaves behind a body of shocked gas which is nonuniform in temperature. Since the ionization rates depend exponentially on this local temperature, there results a corresponding distortion of the ionization profile behind the shock from that which would have prevailed behind a constant strength shock producing an isothermal body of gas. The correction for this effect follows from a straightforward variation of the ionization rate integrals, and is described in detail elsewhere. ${ }^{12}$ It quite

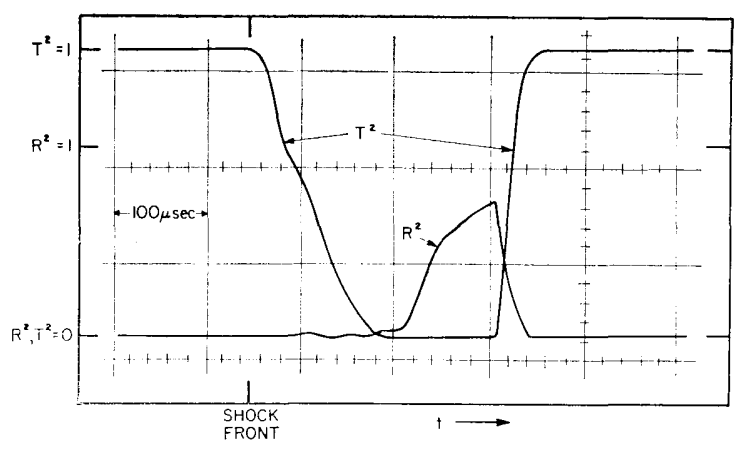

Fig. 3. Typical responses of microwave transmission and reflection detectors to build-up of ionization behind shock front. Total transmission $\left(T^{2}=1\right)$ implies electron densities $n<2 \times 10^{11} \mathrm{~cm}^{-3}$; total reflection $\left(R^{2}=1\right)$ implies $n>$ $10^{13} \mathrm{~cm}^{-3}$.

${ }^{12}$ K. E. Harwell, Ph.D. Thesis, California Institute of Technology (1963). 
frequently altered the raw $n(t)$ data by a large fraction, and is regarded as the principal source of uncertainty in the results. In the worst cases, the $1 \%$ intrinsic inaccuracies in the response of the thin film gauges could be amplified to a $20 \%$ uncertainty in the ionization rate. Fortunately, this appears as a modest random scatter on the semilog data plots, rather than a systematic error which would directly bias the determination of the activation energies.

\section{EXPERIMENTAL RESULTS}

\section{A. Argon}

The corrected electron density profiles are found to be linear in time after passage of the shock front, over the range of sensitivity of the microwave probe $\left(2 \times 10^{11} \leq n \leq 1 \times 10^{13} \mathrm{~cm}^{-3}\right)$. For shocked gas temperatures in the range from about $5800^{\circ} \mathrm{K}$ to $9600^{\circ} \mathrm{K}$, these linear profiles extrapolate back through the time origin at the shock front within the limit of experimental certainty, thus indicating that the "steady state" of the prevailing reaction (cf. Table I) is attained rapidly ( $<5 \mu$ sec). In a few very low temperature shots, $5400^{\circ} \mathrm{K}<T_{2}<$ $5800^{\circ} \mathrm{K}$, there is evidence of a discernible intercept of the linear rise with the positive time axis, perhaps indicative of a more protracted inception phase, but the marginal sensitivity of the microwave probe to these low electron densities, the very small gradients involved, and the larger influence of impurities in this low temperature regime discourage further study of this possibility by the present experiment.

The absolute rates of ionization are found to be remarkably slow in high purity argon. Figure 4 summarizes the observed linear rates for various shocked gas temperatures $T_{2}$ at a preshock ambient pressure of $5 \mathrm{~mm}$. It is found, for example, that for

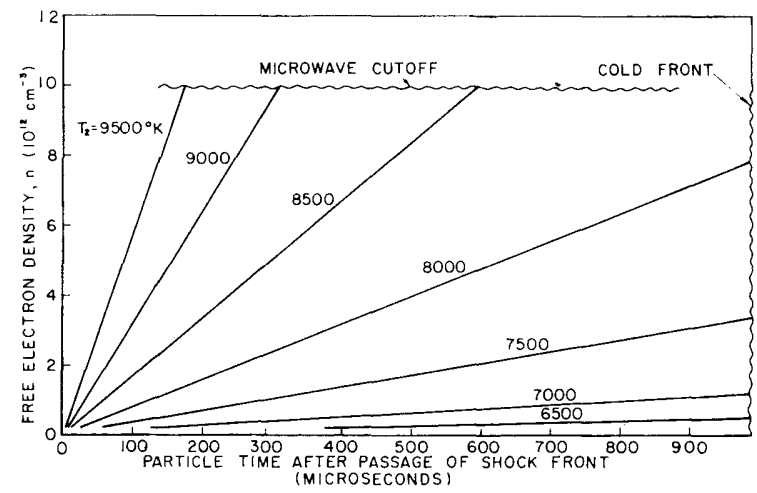

FIG. 4. Ionization profiles behind shock waves in argon.
FIG. 5. Arrhenius plot of ionization rates of shockheated $\operatorname{argon}\left(p_{1}=\right.$ $5 \mathrm{~mm} \mathrm{Hg}$.). Slope: $11.9 \pm 0.5 \mathrm{eV}$

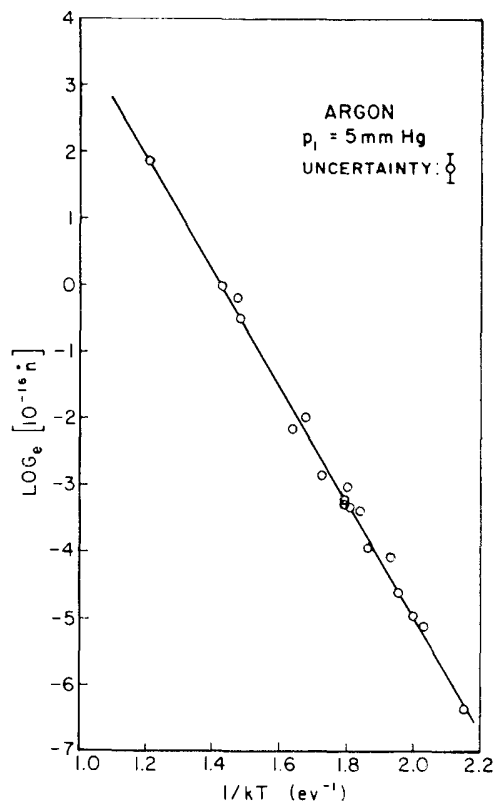

shocks producing temperatures $T_{2} \approx 9000^{\circ} \mathrm{K}$, the theoretically predicted transition to the more efficient electron-atom phase $\left(n>10^{13} \mathrm{~cm}^{-3}\right)$ is reached in about $300 \mu$ sec, particle time. For $T_{2} \approx 8000^{\circ} \mathrm{K}$, over a millisecond is required. In fact, for temperatures lower than $8200^{\circ} \mathrm{K}$, the transition value is not reached in this shock tube before the arrival of the contact front terminates the hot flow. As an extreme case, for a temperature low enough that the equilibrium electron density lies below the transition value $\left(T_{2}<5500^{\circ} \mathrm{K}\right)$, it would take about one second to reach that level via this atom-atom route.

The effective activation energy is obtained from the slope of the graph of $\ln \left(d N_{+} / d t\right)$ vs $(K T)^{-1}$, shown in Fig. 5 for $p_{1}=5 \mathrm{~mm}$. Using a straight line fit to the data points, and iterating through the weak coefficient of relation (10) at $K T=\frac{2}{3} \mathrm{eV}$, the value is $11.9 \pm 0.5 \mathrm{eV}$. This coincides, within the experimental uncertainty, to the first excitation potentials of argon, $11.55-11.83 \mathrm{eV}$, and differs distinctly from the ionization potential, $15.75 \mathrm{eV}$, thereby indicating that the ionization proceeds by a two-step mechanism involving negligible collisional deexcitation or radiative decay (cf. Table I).

Attempts to verify the quadratic density dependence appropriate to this reaction mechanism have been only modestly successful. The range of ambient pressures accessible to this experiment is restricted by the attainable shock strengths and by the microwave sensitivity belt to less than one order of magnitude. Consequently, the experimental un- 


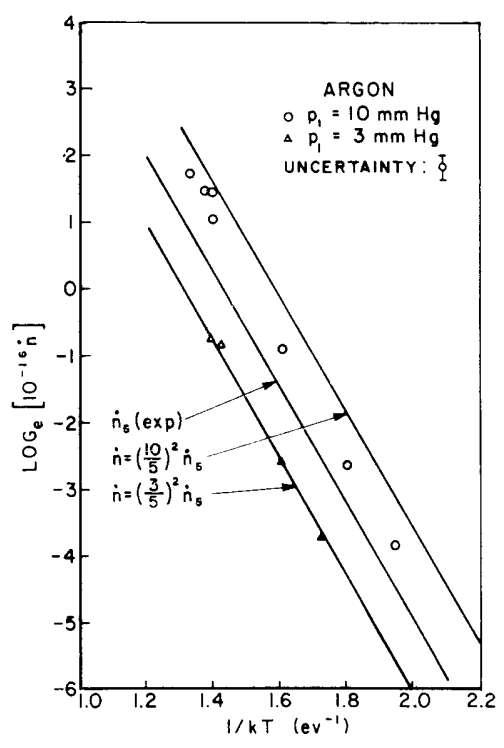

certainties associated with shock attenuation, gas impurity, and the microwave compensations which could be tolerated in the determination of the strong exponential temperature dependence of the ionization rate, become much more troublesome in the study of a mild density dependence. Typical results are displayed in Fig. 6, where the middle line reproduces the $5 \mathrm{~mm}$ data, traced from Fig. 5, and the other two lines are the $5 \mathrm{~mm}$ data normalized by the square of the indicated pressure ratios. The experimental data for $p_{1}=3 \mathrm{~mm}$ support this quadratic scaling, but that for $p_{1}=10 \mathrm{~mm}$ lie systematically below the corresponding line, in some cases in excess of the estimated experimental uncertainty. Since this departure is in the direction

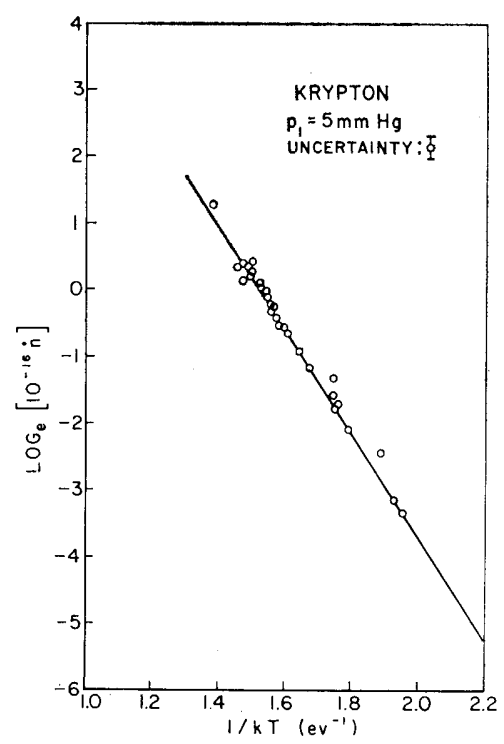

of a lower order density dependence, it favors none of the alternative reactions summarized in Table $\mathrm{I}$.

\section{B. Krypton}

The original purpose of the experiment was a detailed study of the ionization kinetics in argon, and the various techniques involved in obtaining and reducing the data were developed solely with this gas. Only fairly late in the program did it become clear that application of these techniques to another gas would clarify certain of the crucial points in the data reduction, and for this reason, as much as to obtain information on other noble gases, were the studies of krypton, and later xenon undertaken. Since some two or three days are needed to obtain and reduce the data for any one experimental point, less total data was taken for krypton than for argon. By this time, however, the gas handling technique had been well developed, and the overall reliability of the results are felt to be comparable to those for argon.

As in argon, the ionization density in krypton is found to rise linearly in time after passage of the shock over the range of sensitivity of the microwave probe. The absolute rates are somewhat faster than those in argon at the same temperatures, but still very slow on a shock tube scale. For $p_{1}=5 \mathrm{~mm}$, for example, the transition to electron-atom processes would be reached in about $150 \mu \mathrm{sec}$ at $9000^{\circ} \mathrm{K}$, and in about $550 \mu \mathrm{sec}$ at $8000^{\circ} \mathrm{K}$ (particle time).

The graph of $\ln \left(d N_{+} / d t\right)$ vs $(K T)^{-1}$, shown in

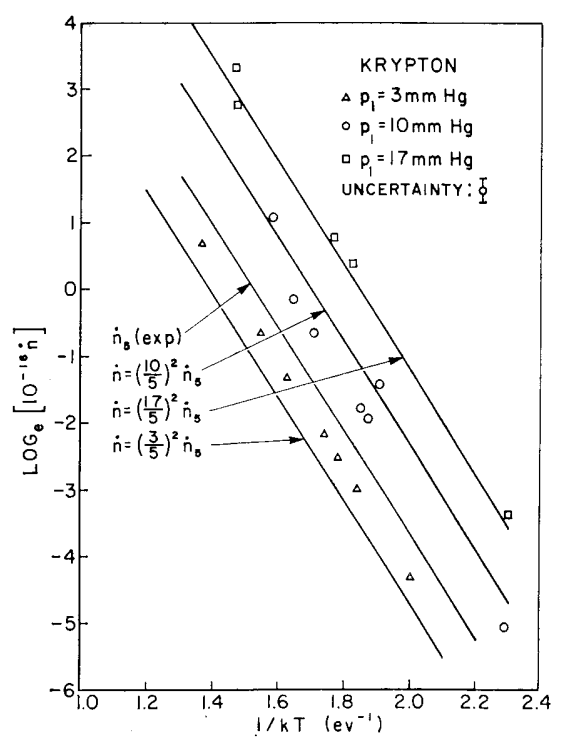

Frg. 8. Pressure dependence of ionization rates in krypton. $3 \mathrm{~mm}, 10 \mathrm{~mm}$, and $17 \mathrm{~mm}$ data superimposed on $5 \mathrm{~mm}$ results $\left(\dot{n}_{5}\right)$, quadratically normalized. 
Fig. 7 yields an effective activation energy of $10.4 \pm 0.5 \mathrm{eV}$, which again overlaps the first excitation potentials $(9.91-10.62 \mathrm{eV})$ rather than the ionization potential $(14.00 \mathrm{eV})$. Thus, the twostep, trapped radiation process again seems to be dominant.

The density dependence of krypton ionization rates is depicted as before in Fig. 8. In this case it is the higher pressure data at $10 \mathrm{~mm}$ and $17 \mathrm{~mm}$ which support a second order dependence, and the $3 \mathrm{~mm}$ data which tend toward a somewhat lower order.

\section{Xenon}

Even less data were taken in xenon, but those taken are felt to be definitive. The ionization again proceeds linearly in time after passage of the shock, at rates slightly higher than those in krypton at the same temperatures. For $p_{1}=5 \mathrm{~mm}$ the electronatom phase is reached in about $140 \mu$ sec at $9000^{\circ} \mathrm{K}$ and about $400 \mu \mathrm{sec}$ at $8000^{\circ} \mathrm{K}$. The graph of $\ln \left(d N_{+} / d t\right)$ vs $(K T)^{-1}$ (Fig. 9) yields an effective activation energy of $8.6 \pm 0.5 \mathrm{eV}$, corresponding again to the first excitation potentials $(8.31-9.55$ $\mathrm{eV})$ rather than the ionization potential $(12.13 \mathrm{eV})$. A very few shots at initial pressures of $3 \mathrm{~mm}$ and $10 \mathrm{~mm}$ again indicated a density dependence slightly weaker than quadratic.

\section{Impurities}

The results described above were obtained using mass spectrometer analysed, "spectroscopic grade" gases continuously circulating through the shock tube in the manner described previously. From comparison of the measured outgassing and flow rates, the maximum impurity level prevailing in these ex-

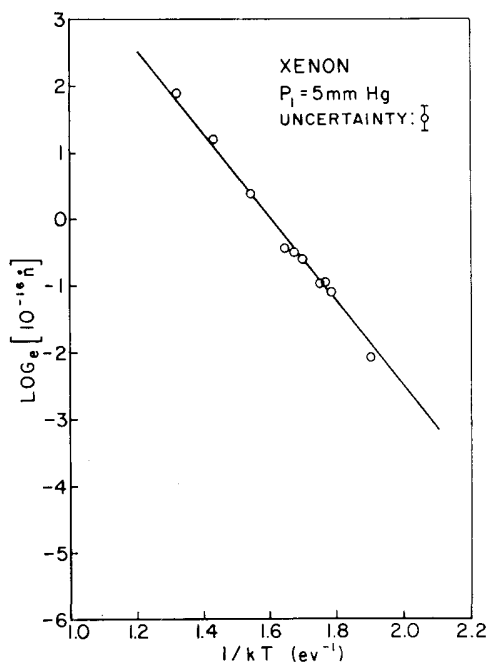

Fig. 9. Arrhenius plot of ionization rates of shockheated xenon $\left(p_{1}=\right.$ $5 \mathrm{~mm} \mathrm{Hg})$. Slope: $8.6 \pm 0.5 \mathrm{eV}$.

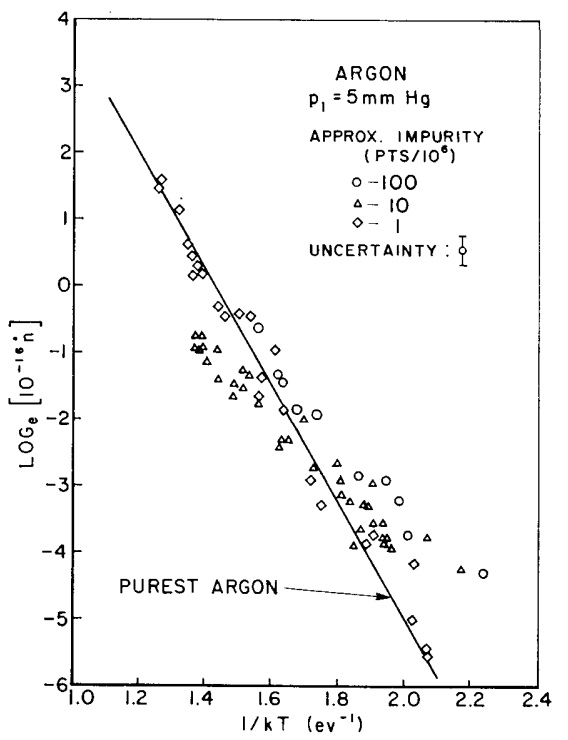

FIG. 10. Effect of impurities on ionization rates of shockheated argon.

periments was a few parts in $10^{7}$. The outgassing species are not known conclusively, but presumably involve some driver gas $\left(\mathrm{H}_{2}\right.$ or $\left.\mathrm{He}\right)$, some water vapor, and indeed, some of the test gas itself, trapped on the walls during a previous circulation process. If the latter were dominant, the net impurity level would be even lower.

Earlier data, obtained with less pure gas samples show significant variations in the absolute ionization rates and effective activation energies down to impurity concentrations of a few parts in $10^{6}$, below which they stabilize on the values displayed above. For example, Fig. 10 superimposes argon ionization rate data taken at various impurity levels on the "pure gas" line of Fig. 5. As might be expected, the low temperature data show progressively higher rates with increasing impurity level. Less anticipated, and as yet unexplained, is the inversion in the high temperature rates, first decreasing, then increasing with increasing impurity level. Of particular significance is the observation that the final improvement in the gas flow purge system, estimated to have lowered the impurity level to a few parts in $10^{7}$, yielded data (solid line) essentially the same as that obtained with the previous system where the impurity level was roughly ten times higher.

A brief study of impurity effects in krypton indicated a more uniform increase in ionization rate with impurity level over the entire range of temperatures studied. No impurity studies were attempted with xenon. 
Clearly, these data permit little conclusion regarding the effect of controlled amounts of given impurities on ionization rates-an interesting problem in its own right. They do emphasize, however, the general requirement for impurity levels below one part per million if significant "pure gas" ionization data are to be obtained.

\section{DISCUSSION AND CONCLUSIONS}

Within the limits of their accuracy, the experiments described above indicate that the initial ionization of shock-heated argon, krypton and xenon proceeds by a two-step atom-atom process

$$
\begin{gathered}
\mathrm{A}+\mathrm{A} \rightarrow \mathrm{A}^{*}+\mathrm{A}, \\
\mathrm{A}+\mathrm{A}^{*} \rightarrow \mathrm{A}^{+}+e+\mathrm{A},
\end{gathered}
$$

where the effective lifetimes of the excited states against radiative decay and collisional de-excitation are sufficiently long to sustain their population at an adequate level to make the first step rate-controlling. The measured activation energies indicate that the predominant excited states involved are the lowest available, namely the electronic configurations $3 s^{2} 3 p^{5} 4 s^{1}$ in argon, $4 s^{2} 4 p^{5} 5 s^{1}$ in krypton, and $5 s^{2} 5 p^{5} 6 s^{1}$ in xenon. Actually, each of these involves four separate levels, two of which are spectroscopically accessible to the ground state and are the origin of the principal resonance lines, and two of which are metastable, with transitions to or from the ground state forbidden by virtue of the $J$ selection rules. ${ }^{13}$ The metastable levels may, however, be accessible by atom-atom collisions, and since the energy separations between them and their allowed neighbors are too small to be distinguished by the above experiments, two alternative interpretations must be admitted: (1) the first atom-atom collision

\footnotetext{
13 "Atomic Energy Levels," Nat. Bur. Std. (U. S.) Circ. 467 (1949).
}

elevates one particle to one of the metastable levels, whose lifetimes are inherently long enough to await a second, ionizing collision; (2) the first collision elevates one atom to one of the spectroscopic resonance levels, whose lifetimes, although much shorter, are effectively protracted by trapping of the resonant photon by adjacent atoms, prior to the subsequent ionizing collision.

In either case, the inception phase for build-up of the excited state concentration to its steady state value is very brief-less than $5 \mu \mathrm{sec}$ for temperatures above $5800^{\circ} \mathrm{K}$. The subsequent linear ionization rate is comparatively slow, ranging from $2 \times 10^{13}$ $\mathrm{cm}^{-3} \mathrm{sec}^{-1}$ at $5500^{\circ} \mathrm{K}$ to $6 \times 10^{16} \mathrm{~cm}^{-3} \mathrm{sec}^{-1}$ at $9500^{\circ} \mathrm{K}$ in argon.

The quadratic density dependence appropriate to this reaction scheme is qualitatively confirmed, although some slightly weaker dependence may be implicit in the data. Unknown impurities in the test gases are found to have profound effects on the ionization rates at concentrations as low as a few parts per million.

\section{ACKNOWLEDGMENTS}

The research described in this paper was supported by the Daniel and Florence Guggenheim Jet Propulsion Center of the California Institute of Technology, and by the U. S. Air Force Office of Scientific Research.

The authors wish to thank Professor F. E. Marble for his many contributions to the design and interpretation of these experiments. Mr. A. J. Kelly assisted with the construction and operation of the equipment, and Mr. H. Wong performed a series of related preliminary studies. ${ }^{14}$ Mrs. B. McAllister carried out many of the numerical computations.

${ }^{14} \mathrm{H}$. Wong, A. E. Thesis, California Institute of Technology (1961). 